Revue d'histoire de l'Amérique française

BEVUE D.HISTOIRE DE L'AMÉRIQUE FRANÇAISE

\title{
CODIGNOLA, Luca et Luigi BRUTI LIBERATI, Storia del Canada, dalle origini ai giorni nostri (Milano, Bompiani, 1999), 815 p.
}

\section{Lucia Ferretti}

Volume 53, numéro 3, hiver 2000

URI : https://id.erudit.org/iderudit/005415ar

DOI : https://doi.org/10.7202/005415ar

Aller au sommaire du numéro

Éditeur(s)

Institut d'histoire de l'Amérique française

ISSN

0035-2357 (imprimé)

1492-1383 (numérique)

Découvrir la revue

Citer ce compte rendu

Ferretti, L. (2000). Compte rendu de [CODIGNOLA, Luca et Luigi BRUTI

LIBERATI, Storia del Canada, dalle origini ai giorni nostri (Milano, Bompiani,

1999), 815 p.] Revue d'histoire de l'Amérique française, 53(3), 429-433.

https://doi.org/10.7202/005415ar d'utilisation que vous pouvez consulter en ligne.

https://apropos.erudit.org/fr/usagers/politique-dutilisation/ 
licisme au monde artistique. Puis en 1926, Maritain, à la demande de Rome, se fait médiateur dans la crise issue de la condamnation de l'Action française de Maurras. L'année suivante, il publie Primauté du spirituel, titre programmatique d'un ouvrage façonné dans la crise. Pour des catholiques qui, comme au Québec, avaient ployé sous le joug de l'ultramontanisme politique et partisan, qui avaient vu le spirituel à l'œuvre dans les élections, l'idée de primauté du spirituel les soulageait de celle de priorité ou de prépondérance.

Maurras avait mis le doigt sur la question sensible en optant pour le «Politique d'abord», lui le non-croyant respectueux des catholiques. Mais comment le catholique pouvait-il suivre ce programme plutôt que celui du «catholique d'abord», de la primauté du spirituel? Comment pouvait-il tenir ensemble «l'universalisme» de la pensée et un certain a-politisme obligé ? Cette posture fut celle des catholiques durant les décennies 1920 et 1930; elle fut au cœur de leurs hésitations, de leurs choix et de leurs aspirations à repenser ces tensions classiques. Et c'est du centre de ce travail volcanique que l'intellectuel catholique peut émerger, et en particulier dans la figure de Maritain.

Il eût été intéressant ici que P. Chenaux fasse le point sur les travaux récents (Fouilloux, Colin, $\mathrm{n}^{\circ} 13$ de Mil neuf cent) à propos de «l'intellectuel catholique » et qu'il inscrive son apport dans ces acquis et ces questionnements. La chose s'imposait d'autant plus que le propos et la conclusion du livre visaient cette question.

Philippe Chenaux avait raison de penser initialement inclure le Québec dans son enquête. On attend toujours l'étude sur le sujet qu'il a couvert. Il y a bien les travaux d'Yvan Cloutier sur le Maritain de 1930 au Québec, mais des études sur la perception québécoise de Maritain dans la trame de la condamnation de l'Action française de Paris (et non de Montréal), point. L'urgence d'une telle analyse s'impose d'autant plus que celle-ci permettrait de comprendre comment se recompose au Québec la question des rapports du spirituel et du temporel. Une telle enquête devrait faire place à la présence d'Étienne Gilson, d'Yves Simon et du médiéviste franciscain québécois Ephrem Longpré, à celle des Dominicains québécois et français (Chenu), à l'influence de Louvain et du chanoine Jacques Leclercq et à la problématique d'un «nouveau Moyen Âge» — plus tardive ici — formulée par le dominicain Benoît Lacroix.

Département de langue et littérature françaises

Université McGill

YVAN LAMONDE

CODIGNOLA, Luca et Luigi BRUTI LIBERATI, Storia del Canada, dalle origini ai giorni nostri (Milano, Bompiani, 1999), 815 p.

Je m'attendais à une synthèse générale de l'histoire du Canada depuis les origines, telle qu'on peut en lire en anglais depuis longtemps, et même en 
français depuis une bonne décennie. Ne pensons qu'à la traduction chez Boréal de l'Histoire générale du Canada, paru sous la direction de Craig Brown, ou à un ouvrage comme celui de Cardin, Couture et Allaire, Histoire du Canada. Espaces et différences. J'ai trouvé en effet une synthèse honnête dans la deuxième partie de la Storia, écrite par Luigi Bruti Liberati; dans la première partie, en revanche, Luca Codignola se livre à un remaniement si considérable de la mémoire historique des Canadiens que l'entreprise paraît obéir à des fins politiques plus que scientifiques.

Bompiani, éditeur milanais, s'est lancé dans une collection d'«histoire du monde en poche». Déjà plusieurs volumes ont paru, sur l'histoire de la Yougoslavie, celle de la Russie, des États-Unis, de l'Espagne et de quelques autres pays. Aucun de ces livres n'a été écrit par des historiens italiens; sauf ceux sur la Grèce, et peut-être Israël, tous au contraire ont été rédigés par des ressortissants des pays concernés, puis traduits. Ce qui fait de l'ouvrage sur le Canada une entreprise non seulement unique pour l'instant dans la collection, mais a priori très intéressante puisque voilà une occasion, pas si courante après tout, de parcourir l'ensemble de l'histoire de ce pays et de ses habitants à travers les yeux de spécialistes étrangers.

Pour rassembler les matériaux de leur ouvrage, subventionné par le ministère des Affaires extérieures du Canada, les auteurs ont privilégié l'historiographie canadienne des derniers dix ans, qu'ils ont abondamment consultée, dans les deux langues. Ils ont néanmoins bâti leur synthèse surtout à partir de l'historiographie écrite en anglais. Celle-ci, en effet, ne sert pas seulement à documenter des questions spécifiques, comme c'est l'usage généralement fait de l'historiographie rédigée en français; les historiens canadiens-anglais inspirent aussi aux auteurs, surtout à Codignola, leur manière de poser les problèmes généraux. William Eccles est largement utilisé pour la Nouvelle-France et les ouvrages de John Dickinson et Brian Young sont présentés comme «i punti di partenza» (les points de départ) d'une découverte du Québec; ceux auxquels, ensemble ou séparément, ont contribué notamment David J. Bercuson et Jack Granatstein reviennent souvent eux aussi. La bibliographie recense également la production sur l'histoire du Canada écrite en italien, qui apparaît somme toute assez riche et diversifiée.

Les auteurs ont voulu écrire une histoire à la fois rigoureuse et accessible. Leur plan très systématique, divisé selon une démarche analytique, sait se combiner avec une rédaction de type narratif dans laquelle les acteurs et les événements, toujours individualisés, sont en même temps inscrits dans les tendances générales de l'histoire que les auteurs ont choisi de dégager. Codignola enseigne l'histoire nord-américaine à l'Université de Gênes; son regard sur le Canada est toujours continental. Bruti Liberati enseigne l'histoire contemporaine à l'Université de Milan: il sait que les rapports de force entre nations existent, et colorent non seulement les relations internationales mais aussi les rapports internes.

Comment résumer cette Storia del Canada? J'en dégagerai plutôt les articulations principales. 
Bruti Liberati couvre à lui seul la période de 1867 à nos jours. Il choisit de la narrer selon trois angles bien distincts, et d'inégale longueur: «Le Canada et le monde», "La Confédération canadienne», «Les Italiens au Canada». Chacune de ces sections suit l'ordre chronologique. Sans doute, les canadianistes n'apprendront pas beaucoup de cette histoire somme toute classique. Le mérite incontestable de Bruti Liberati est toutefois d'avoir réussi à présenter à un public non spécialiste, Italiens d'Italie et Italiens d'ici, une histoire centrée sur la mise en perspective historique de quelques grands enjeux contemporains.

Par exemple, la difficulté pour le Canada d'élaborer, et même simplement de vouloir, une politique étrangère autonome. Bruti Liberati situe bien le contexte: la dépendance coloniale au sein de l'Empire britannique, le plus puissant du monde; puis la dépendance économique et militaire à l'égard des États-Unis; les tensions internes parfois explosives, qu'il n'hésite pas à reconnaître comme des tensions «nationales », depuis la guerre des Boers à celle des drapeaux, sous la Révolution tranquille. Bruti Liberati sait aussi faire ressortir les rôles d'un pionnier comme Henri Bourassa puis de deux premiers ministres libéraux, Mackenzie King et Trudeau, dans les brefs moments où le Canada a tenté de faire entendre une voix plus personnelle sur la scène internationale. Il sait enfin, quoique sans le souligner à gros traits, faire prendre conscience aux lecteurs combien, depuis une bonne trentaine d'années, le Canada semble satisfait de son alignement automatique sur la politique extérieure des États-Unis, et combien au Québec même, chose nouvelle, presque plus personne, et surtout pas le gouvernement, ne conteste les orientations internationales suivies par Ottawa.

La section la plus considérable de cette seconde partie de la Storia porte sur l'évolution interne de la Confédération. Ici encore, l'accent est mis délibérément sur l'histoire politique. Car il faut dire qu'une place très congrue est réservée dans cette synthèse à l'histoire culturelle, et une autre assez mince à l'histoire sociale, même si les respirations et les contractions de l'économie sont bien présentes, ainsi que leurs conséquences sur le mouvement ouvrier ou sur l'expression d'idéologies de gauche comme de droite. Ce que veut surtout Bruti Liberati, c'est faire comprendre à ses lecteurs les grands axes de la vie politique canadienne. Il démêle devant eux l'écheveau complexe de la domination nationale des Canadiens anglais sur les Premières Nations et sur les Canadiens français, celui des revendications constitutionnelles des provinces et notamment du Québec, et souligne le projet fédéral des trois dernières décennies de redéfinir l'identité canadienne autour de la promotion du multiculturalisme. Son histoire, qui conduit réellement jusqu'à nos jours, fait la part des blocages, des aspirations déçues et des luttes qui font le Canada politique actuel. Bruti Liberati finit cette section sur une note un peu NPD: que son avenir soit avec ou sans le Québec, écrit-il, le Canada, qui se caractérise par ses programmes sociaux, par le rôle des syndicats et par une faible violence (respect de la loi, abolition de la peine de mort) restera une réelle solution de rechange nord-américaine aux États-Unis. 
Le court chapitre qui clôt l'ouvrage met surtout l'accent sur les vagues migratoires qui ont conduit 700000 Italiens au Canada entre 1861 et 1981, et sur leur progressive insertion dans la société canadienne. Les travaux de Robert Harney, ceux de Roberto Perin et de Bruno Ramirez, entre autres, permettent de retracer à grands traits les évolutions de la vie communautaire, encore qu'une fois de plus, la préoccupation de Bruti Liberati se révèle plus politique que sociale. La vitalité culturelle récente de la communauté italienne de Toronto et de Montréal est également soulignée.

Luca Codignola s'est chargé de toute la partie de l'histoire du Canada qui précède la Confédération. Il a choisi de le faire d'une manière nettement moins classique que son collègue mais, à mon sens, malheureusement nettement moins crédible aussi.

Codignola écrit en quelque sorte l'histoire d'un espace géographique: celui que définissent les frontières politiques actuelles du Canada. Ses objectifs sont nombreux. Le premier: montrer que dès l'origine, soit dès le passage des Asiatiques par le détroit de Behring il y a 20000 ans, le Canada est un pays d'immigrants et une société multiculturelle. Le second: ôter toute légitimité à la prétention des Canadiens français de former une nation. Le troisième: montrer que c'est par une recherche constante — et résolue dans l'harmonie - d'équilibre entre des régionalismes potentiellement centrifuges et des exigences de la collaboration à l'échelle continentale que le Canada a pris, dans la première moitié du $\mathrm{XIX}^{\mathrm{e}}$ siècle, les traits qui allaient définir sa personnalité «nationale», incarnée d'abord dans l'Union, puis dans la Confédération.

Chacun de ces objectifs est poursuivi avec constance tout au long des 450 pages qui forment la première partie de la Storia. Parmi les points forts de cette section, l'inscription de l'espace géographique canadien dans l'ensemble de l'Amérique du Nord, un réel souci de présenter l'histoire du point de vue amérindien, une attention plus grande que chez Bruti Liberati pour l'économie et pour la société (les femmes, cependant, sont très peu présentes dans cette Storia). Et surtout, un exposé d'une qualité pédagogique remarquable. Toutes les idées d'un chapitre sont annoncées d'entrée, elles sont ensuite développées l'une après l'autre en étant bien identifiées, des conclusionsbilans ramassent aussi toujours le propos. Les lecteurs ne sont ainsi jamais livrés à eux-mêmes, ce qui devrait contribuer au succès de l'ouvrage dans le grand public.

Mais l'ouvrage comporte aussi des faiblesses. Je soulignerai surtout celles qui m'ont le plus irritée, et qui concernent le traitement de la question des « francophones».

Aucune des dates charnières de l'histoire de la Nouvelle-France, telle que les historiens ont l'habitude de la raconter, n'est retenue comme significative dans cette partie. Ni 1534-1535 (Jacques Cartier est mentionné en note infrapaginale), ni 1608, ni même 1759-1760. Des Acadiens (ou plutôt de la «communità di origine acadiana»), les lecteurs retiendront surtout qu'une majorité d'entre eux parle micmac à la maison (p. 47), qu'ils sont déchirés au 
XVII ${ }^{e}$ siècle par une guerre civile entre deux clans familiaux et que, s'ils avaient comparé leur déportation à ce qui se passait au même moment sur les champs de bataille européens, «essi si sarebbero potuti dire fortunati» (ils auraient pu se dire qu'ils étaient chanceux) (p. 226). Les francophones du Québec, pour leur part, forment sous le Régime français une société qui est décrite essentiellement à partir du témoignage que le gouverneur Murray a laissé d'eux. Les groupes sociaux entre lesquels ils se séparent ne forment jamais un peuple, mais au mieux une entité distincte de la métropole. L'Église, qui a assuré la survie de la colonie (p. 70), a fait de la religion un trait distinctif de la mentalité française coloniale. La Conquête n'a que peu de conséquences, car les élites s'en vont, et ceux qui restent l'acceptent, y compris l'Église. D'ailleurs le statut de celle-ci est meilleur après qu'avant (p. 265) et elle s'impose vite et durablement comme le seul définisseur de la culture canadienne-française. Du reste, la recherche du compromis, caractéristique de l'histoire du Canada, s'est faite «senza traumi e conflitti maggiori» (sans traumatisme ni conflit majeur) durant la première moitié du $\mathrm{XIX}^{\mathrm{e}}$ siècle, «quand'anche si considerino le Ribellioni del 1837-1838 » (même si l'on considère les Rébellions) (p. 343). Le régime de l'Union a été un élément d'unification de l'Amérique du Nord britannique (p. 325) et il a préparé les Canadiens à désirer la Confédération.

Il y a bien sûr autre chose que cela dans la première partie de la Storia, mais ce côté d'histoire à l'eau de rose est profondément déplaisant. On est loin des National Standards for United States History, qui font le pari que la société américaine résoudra d'autant mieux ses conflits internes contemporains qu'elle aura mieux posé ceux qui l'ont traversée au cours de l'histoire. Le parti pris trop visible de Codignola pour la bonne-entente finit par nuire à son propos.

Département des sciences humaines

Université du Québec à Trois-Rivières

LUCIA FERRETTI

COLLARD, Chantal, Une famille, un village, une nation. La parenté dans Charlevoix, 1900-1960 (Montréal, Boréal, 1999), 194 p.

Dans ce livre, Chantal Collard montre l'importance de la parenté dans la société rurale du Québec d'avant 1960. Le terrain d'enquête est un village de Charlevoix désigné sous le nom fictif de Cap-Saint-Michel. Cependant, à partir de ce cas, l'auteure entend saisir une culture rurale de la parenté dépassant largement les limites de Charlevoix. Elle prend donc soin de relever, à chaque étape de sa démonstration, les nombreux éléments comparatifs avec divers autres travaux d'anthropologues, de sociologues et d'historiens. Cet ouvrage est le produit d'une patiente enquête ethnologique sur le terrain durant laquelle l'auteure a interrogé de nombreuses personnes dont certaines étaient, comme elle, des passionnées de la parenté. Cette enquête est complétée par l'examen des archives locales et la saisie informatisée des données 